\title{
Factors associated with active aging in Chinese elderly in rural areas of Xiangtan County, China ${ }^{\dagger}$
}

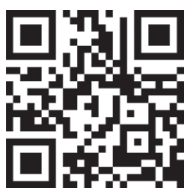

Original article

\author{
Xue-Lian Fua, Jian-Guang Lia, Yin-Li Sua, Hong-Hong Wang ${ }^{b}$, Yang Guoa, Li-Qiong Liua, \\ Chun-Yan Zeng ${ }^{a}$
}

aXiangtan Medicine and Health Vocational College, Xiangtan, Hunan 411104, China

${ }^{b}$ School of Nursing, Central South University, Changsha, Hunan 410013, China

Received: 12 April 2021; Accepted: 20 May 2021; Published: 20 December 2021

Abstract: Objectives: To explore the level of active aging and correlates among rural elderly in Xiangtan County, China. Methods: A cross-sectional survey was conducted from July to November 2019. A total of 945 rural elderly from 3 towns in Xiangtan County, China, were investigated by using the positive aging evaluation questionnaire (PAEQ), the activity of daily living scale (ADL), and the depression in old-age scale (DIA-S).

Results: The average score in the PAEQ among the rural elderly of Xiangtan County was 72.81 (range $=21-105$ ). A moderate negative correlation was found between the score in the PAEQ and those in the ADL $(r=-0.361)$ and DIA-S $(r=-0.495)$. Symptoms of depression ( $\beta$ : $-0.321, P=0.001)$, number of chronic diseases $(\beta:-0.281, P=0.001)$, subjective economic status ( $\beta$ : 0.239 , $P=0.001)$, ADL ( $\beta:-0.196, P=0.001)$, education $(\beta: 0.126, P=0.001)$, number of children $(\beta: 0.097, P=0.001)$, and marital status $(\beta$ : $-0.060, P=0.001)$ were significantly associated with levels of active aging in rural elderly, whereas gender and age were not independently related to active aging.

Conclusions: The level of active aging is at a moderately high level in the Chinese elderly in rural areas in Xiangtan County. The focus group of active aging in rural areas should be elderly people suffering from chronic diseases, physical decline, poor education and economic conditions, childlessness, and those without partners.

Keywords: elderly • active aging • rural area

(c) Shanxi Medical Periodical Press.

\section{Introduction}

Aging has become a global trend. By $2050,22 \%$ of the world's population will be over 60 years old, which is an increase from $11 \%$ in 2019 . With the rapid increase of the aging population, a series of social problems brought about by the older population, such as health concerns and support and nursing for the aged, is already causing a wide social concern. Positive and effective measures to cope with these problems must be taken and prioritized worldwide.

If everyone can achieve a relatively close life expectancy and healthy lifespan and can spend their old age independently, then the problem of aging can be effectively solved. Under the guidance of this concept, the World Health Organization (WHO) introduced the

† This project was supported by Hunan Social and Social Science Achievement Review Committee (No. XSP19YBC050) and Hunan Provincial Health Commission (No. 20200094).

How to cite this article: Fu XL, Li JG, Su YL, et al. Factors associated with active ageing in Chinese elderly in rural areas of Xiangtan County, China. Front Nurs. 2021;4:407-418. 
concept of active aging, which is sometimes translated as "positive aging". Active aging has been defined as "... the process of optimizing opportunities for health, participation, and security, in order to enhance quality of life and well being as people age". ${ }^{1}$ It is an umbrella concept embracing a semantic space in which healthy, successful, and productive aging are strongly related. ${ }^{2}$ Unlike other theories of aging, active aging puts the elderly in an active position in the process of aging rather than in a passive one. It also includes old men suffering from any disability or disease. It encourages the elderly to participate in various social and family activities, which plays an important role in enhancing the self-worth of the elderly, maintaining individual physiological functions, and strengthening inter-generational friendship and solidarity. Importantly, the realization of active aging of the elderly can greatly reduce the expenditure of social care. Moreover, by making the best use of human capital and increasing community participation and mutual assistance, active aging also plays an important role in maintaining social stability and developing the economy. ${ }^{3}$ The United Nations Madrid II International Plan of Action on Aging adopted active aging and inspired policies at national, regional, and international action. "Report on ageing and health" also emphasizes the important position of active aging policy in the process of coping with the aging society. ${ }^{4}$ Active aging has even been converted into a mantra in aging societies. ${ }^{2}$

The mining and analysis of the influencing factors of active aging can provide a basis for the realization of active aging on a personal level. The WHO has initially formed a model of active aging determinants, including health and social services (health promotion and disease prevention, curative service, long-term care, mental health services), behavioral factors (tobacco use, physical activity, healthy eating, oral health), personal factors (biology and genetics, psychological factors), physical environment (physical environments, safe housing, falls, clean water, clean air, and safe food), social environment (social support, violence and abuse, education, literacy), and economic factors (income, social protection, work), which are embedded in cultural and gender contexts. ${ }^{1}$ This model has aroused much attention and controversy among scholars. ${ }^{5}$ Many of the determinants proved to be entwined, reflecting the transaction between individual and environmental factors in shaping adaptation to the aging process. ${ }^{6}$ Some of these determinants are policy issues that need to be actively addressed by the government, such as the promotion and integration of health and social services and the construction of suitable aging environments. Some factors have been demonstrated by many studies as good, healthy behavior, and the construction of an aging environment will inevitably make active aging easier to achieve.

Gender and age differences affect the active aging situation. ${ }^{7}$ The younger the elderly, the higher the level of active aging. ${ }^{8-10}$ However, the effect of gender on active aging is controversial. ${ }^{2,11}$ Regarding physical environment and economic factors, a high level of education, ${ }^{8,9,12,13}$ good economic condition, ${ }^{8,14}$ the absence of chronic disease, ${ }^{8,14,15}$, and abundant family support ${ }^{15}$ are linked to high active aging levels. However, specific results vary across studies in different populations.

Good daily living ability and the absence of depressive symptoms have also been classified as indicators of active aging. ${ }^{11}$ Pham et al. found that active aging is positively associated with perceived health, life satisfaction, and quality of life and negatively associated with falls, loneliness, and frailty. ${ }^{16} \mathrm{~A} 6$-factor model (i.e., health, psychological component, cognitive performance, social relationships, bio-behavioral component, and personality) was formed through interviews with 1322 old people living in the community. Findings reinforced the importance of psychological functioning in active aging in the oldest old. ${ }^{6,17}$ Active aging is strongly consistent with low depressive symptoms. ${ }^{18}$

However, due to transportation, language, funding, and other obstacles, the research and promotion of active aging are mostly concentrated in urban areas, involving very few in rural areas. Furthermore, inequalities in the development of health care and social services are apparent among Western countries, ${ }^{19}$ African countries, ${ }^{20}$, and China. ${ }^{21}$ The situation of the elderly in rural areas requires more attention.

Therefore, this study aimed to explore the level and factors associated with active aging in rural areas of Xiangtan County, China, and to provide health management suggestions to the health service sector for the rural elderly.

\section{Methods}

\subsection{Design}

This research involved a cross-sectional and descriptive study conducted in rural areas of Xiangtan County, China.

The research site of this study was located in rural areas of Xiangtan County, Hunan Province, Southeast China. According to the 2018 national economic and social development statistics bulletin, the total number of people in Xiangtan is 2.865 million, of which 0.61 million $(21.3 \%)$ were aged 60 years and older. ${ }^{22}$ The average life expectancy of the Xiangtan area is 78.63 years, which is higher than the national and provincial average 
levels. In summary, aging is a very important and burning social realistic issue in the region.

Considering previous data showing $36.67 \%$ satisfaction of rural elderly pension status ${ }^{23}$ and according to the formula $\mathrm{n}=\left(\mathrm{Z}_{\alpha / 2}\right)^{2} \times P(1-\mathrm{p}) / \delta^{2}$, taking $\alpha=0.05$, $Z_{\alpha / 2}=1.96, \delta=10 \% \times P$, and $P=37 \%$, the sample size was 654 cases. Considering the possible loss of samples, the sample size was increased by $20 \%$. Hence, the sample size should be at least 785 cases.

Multistage sampling was adopted. First, according to the per capita annual income of farmers, 17 towns in Xiangtan County were divided into 3 levels: good, medium, and poor. In each level, a township was selected by the random number method. Three towns were selected: Shitan Town (2220 USD per year), Fenshui Town (1855 USD per year), and Zhonglupu Town (1609 USD per year). In each town, 3 villages were selected by random number simple sampling. Finally, 9 administrative villages were selected as the source of the survey objects.

Old adults were recruited in the study if they (1) were aged 60 years or over, (2) continuously lived in the local area for $>1$ year, and (3) were clear-minded and able to communicate effectively. Residents in hospitals or nursing homes were excluded from the survey.

\subsection{Measurements}

\subsubsection{General information sheet}

A general information sheet was used to collect demographic and general health data, including gender, age, education, marital status, living arrangement, number of children, subjective economic status, and number of chronic diseases.

\subsubsection{Assessment of active aging}

The positive aging questionnaire (PAEQ) compiled by Hu Min et al. in 2012 was used to evaluate the active aging level. The questionnaire was developed based on the concept of active aging and its three pillars, with the aim of developing an individual-level instrument for measuring active aging. The overall Cronbach's $\alpha$ coefficient of the questionnaire was 0.914 . The overall semi-reliability of the questionnaire was 0.801 . The content validity of the questionnaire was analyzed by the expert judgment method, and the Content Validity Index (CVI) was 1. The correlation between each dimension and the total questionnaire ranged from 0.137 to 0.888 , and the construct validity was good in Hu's study. ${ }^{24}$ The overall Cronbach's $\alpha$ coefficient of the questionnaire in this study was 0.862 . The assessment includes physical vitality (i.e., the physical activity of the elderly), life satisfaction (i.e., the spiritual and cultural aspects of the elderly and the status of their own life satisfaction), family support (i.e., family members, friends, or social groups to support and help the elderly), and active participation (i.e., the participation of the elderly in social activities and social services) in 4 dimensions. The assessment included 21 items that involved the use of a 5-point scoring method from "completely inconsistent" (1 point) to "fully qualified" ( 5 points); the median number is 3 . Entries with scores of $\leq 1, \geq 2$ but $<3, \geq 3$ but $<4$, and $\geq 4$ indicate low, moderately low, moderately high, and high scores, respectively. The total score may range from 21 to 105 points, with scores of $\leq 42,>42$ but $\leq 63,>63$ but $\leq 84$, and $>84$ indicating low, medium-low, medium-high, and high levels of active aging, respectively. A high score indicates a high level of active aging.

\subsubsection{Assessment of activities of daily living}

Activities of daily living were measured using the activity of daily living scale (ADL) developed by Lawton and Brody in $1969 .{ }^{25}$ It consists of the physical self-maintenance scale and the instrumental activities of daily living scale. The first part includes 6 items, namely, walking, eating, dressing, grooming, bathing, and toilet use. The second part is an instrumental daily living ability scale that consists of 8 items, namely, using transportation, cooking, doing housework, taking medicine, doing laundry, shopping, using of phone, and administration of their economy. Choices for each item were graded "1 = can do it myself," "2 = have some difficulty doing," "3 = need help to do it," and " 4 = cannot do it at all". The total score of ADL represents the different dependence degrees of the elderly: $\leq 14$ indicates the normal ability of daily living, whereas $>14$ indicates partial limitation of the ability of daily living. The Cronbach's $\alpha$ of ADL was 0.918 in this study.

\subsubsection{Assessment of depressive symptoms}

The depression in old-age scale (DIA-S) was used to measure symptoms of depression. It was compiled by Heidenblut and Zank, designed specifically for the elderly, ${ }^{26}$ and has been used in Germany and Iran. ${ }^{27,28}$ The scale included 10 depression-related items that were to be answered with true or false with a yes/no answer format. The scale ranges from 0 (no depressive symptoms) to 10 (maximal number of depressive symptoms). The total maximum score is 10 . A cut-off of $\geq 3$ was considered an indication of depressive symptoms. High scores suggest high levels of severe depressive symptoms. Yang Bing et al. ${ }^{29}$ produced a translated 
version of the DIA-S in Chinese. The Chinese DIA-S has a total Cronbach's coefficient of 0.829 , a split-half reliability of 0.831 , a retest reliability of 0.907 and a content validity index (S-CVI/UA) of 0.9 . The content validity index (I-CVI) of item level was $0.833-1$. The average content validity index (S-CVI/Ave) was 0.983 , and the Pearson correlation coefficient between each entry and the total score was $0.475-0.789$. The coefficient of the internal consistency of the scale was 0.787 in this study.

\subsection{Procedure of data collection}

After unified training and assessment, 3 investigators entered the home of the elderly in the countryside and conducted face-to-face investigations with the assistance of the staff in each village. Given the common characteristics of poor eyesight and low education of the elderly in rural areas, the survey was conducted in the form of face-to-face interviews, and all data were recorded by investigators. Approximately 20-30 min were taken to complete a questionnaire. After the investigations were concluded, respondents were thanked for their participation and given some incentives, such as a pack of food. The data collectors performed the necessary checking and verification procedures in time after the participants had completed the questionnaire.

\subsection{Data analysis}

Information from the questionnaires was typed into a computer by 2 researchers according to the serial numbers. SPSS 19.0 was used for data analysis. Categorical variables (gender, age, education, marital status, number of children, subjective economic status, and number of chronic diseases) were presented as frequencies and percentages, whereas continuous variables (positive aging level, activities of daily living, and depression symptoms) were expressed as mean \pm standard deviation (SD). Self-care dependence and depressive symptoms in the elderly were also measured as percentages. A single sample $t$-test was used to compare the mean scores of PAEQ between the study and that of Cao's. ${ }^{12}$ Independent sample $t$-test and ANOVA were applied to examine the difference in active aging level among sociodemographic variables.

Correlations between PAEQ score, ADL, and depressive symptoms were analyzed using Pearson's correlation analysis. Multiple linear regression was used to explore the influential factors affecting the level of active aging of rural elderly. In the study, $P$-values $<0.05$ were considered significant.

\section{Results}

\subsection{General characteristics of the participants}

A total of 945 rural elderly were investigated, 10 of which stopped answering due to time shortages during the investigation. Finally, 935 valid questionnaires (98.9\%) were collected. Among the participants, 477 (51.0\%) were males, and 437 (46.8\%) participants were aged 60-69 years. Most of the participants had primary education or below $(76.8 \%, \mathrm{n}=718)$, married and with a living spouse $(70.8 \%, \mathrm{n}=662)$ and had $2-3$ children $(68.8 \%$, $n=643)$. With regard to financial status, $477(51 \%)$ respondents reported that they were in a fair financial status. In terms of health, $511(54.6 \%)$ participants suffered from 2 or more types of chronic diseases, as shown in Table 1.

\subsection{Descriptive analysis of activities of daily living}

The results showed that $44.7 \%(n=418)$ of the elderly suffered from impaired activity of daily living. A higher number of older adults were impaired in instrumental daily living ( $n=412,44.1 \%)$ than in self-maintenance ability ( $n=177,18.9 \%)$, as shown in Table 2 .

\subsection{Symptoms of depression in the elderly in rural areas}

Of those surveyed, $19.7 \%(n=184)$ reported symptoms of depression. Furthermore, $29.5 \%(n=276)$ of older people found difficulty in relaxing, whereas $23.2 \%(n=217)$ recently (nearly 2 weeks) felt spiritless (Table 3 ).

\subsection{Levels of active aging and its associations with demographic characteristics}

A descriptive analysis of active aging is presented in Table 4. The average score of active aging among the rural elderly was 72.81 , which belongs to the medium upper level.

The mean scores of the 4 dimensions (physical vitality, life satisfaction, family support, and active participation) of PAEQ were 3.28 (SD $=0.98$, scale range $1-5)$, $3.70(\mathrm{SD}=0.78$, scale range $1-5), 4.40(\mathrm{SD}=0.94$, scale range $1-5)$, and 2.81 ( $S D=0.95$, scale range 1-5), respectively. The total score and dimensions of life satisfaction and physical vitality were significantly lower than those in Cao's findings ${ }^{12}(P<0.001)$.

The results of the correlation analysis among the independent variables and active aging are summarized in Table 5. Significant differences in active aging 


\begin{tabular}{|c|c|c|}
\hline Variables (theoretical ranges) & $n$ & $\%$ \\
\hline \multicolumn{3}{|l|}{ Gender } \\
\hline Male & 477 & 51.0 \\
\hline Female & 458 & 49.0 \\
\hline \multicolumn{3}{|l|}{ Age } \\
\hline $60-69$ & 437 & 46.8 \\
\hline $70-79$ & 381 & 40.7 \\
\hline$\geq 80$ & 117 & 12.5 \\
\hline \multicolumn{3}{|l|}{ Education } \\
\hline Primary school or below & 718 & 76.8 \\
\hline Junior school & 166 & 17.7 \\
\hline High school & 42 & 4.5 \\
\hline College or higher & 9 & 1.0 \\
\hline \multicolumn{3}{|l|}{ Marital status } \\
\hline $\begin{array}{l}\text { Married and with a } \\
\text { living spouse }\end{array}$ & 662 & 70.8 \\
\hline Single or widowed & 266 & 28.5 \\
\hline Divorced or separated & 7 & 0.7 \\
\hline \multicolumn{3}{|l|}{ Number of children } \\
\hline 0 & 40 & 4.3 \\
\hline 1 & 94 & 10.0 \\
\hline $2-3$ & 643 & 68.8 \\
\hline$\geq 3$ & 158 & 16.9 \\
\hline \multicolumn{3}{|l|}{ Subjective economic status } \\
\hline Poor & 318 & 34.0 \\
\hline Fair & 477 & 51.0 \\
\hline Good & 140 & 15.0 \\
\hline \multicolumn{3}{|l|}{ Number of chronic diseases } \\
\hline 0 & 194 & 20.8 \\
\hline 1 & 230 & 24.6 \\
\hline $2-3$ & 362 & 38.7 \\
\hline$\geq 3$ & 149 & 15.9 \\
\hline
\end{tabular}

Table 1. Characteristics of participants $(N=935)$.

were found for gender $(t=2.404, P=0.016)$, education $(F=15.348, P<0.001)$, marital status $(F=13.687$, $P<0.001)$, number of children $(F=11.484, P<0.001)$, subjective economic status $(F=73.227, P=0.001)$, and number of chronic diseases $(F=61.257, P<0.001)$. Furthermore, the elderly who were male, married and with living spouses, accepted higher education, had two to three children, had good subjective economic status, and had no chronic disease were more likely to be in a higher level of active aging. However, we did not find a

\begin{tabular}{lll}
\hline Dimensions (Score ranges) & $n$ & $\%$ \\
\hline \hline Physical self-maintenance & & \\
Independent (=6) & 758 & 81.1 \\
Dependent (>6) & 177 & 18.9 \\
Instrumental activities & & \\
Independent (=8) & 523 & 55.9 \\
Dependent ( $>8)$ & 412 & 44.1 \\
Total score of ADL & & \\
Independent $(=14)$ & 517 & 55.3 \\
Dependent $(>14)$ & 418 & 44.7 \\
\hline
\end{tabular}

Note: $\mathrm{ADL}$, activity of daily living.

Table 2. ADL of participants $(N=935)$

\begin{tabular}{|c|c|c|}
\hline Items & $n$ & $\%$ \\
\hline 1. I've been feeling down lately. & 217 & 23.2 \\
\hline $\begin{array}{l}\text { 2. I'm worried about saying or doing something } \\
\text { wrong. }\end{array}$ & 50 & 5.3 \\
\hline 3. It's hard for me to relax. & 276 & 29.5 \\
\hline 4. My life seems meaningless. & 59 & 6.3 \\
\hline 5. I have a hard time motivating myself. & 147 & 15.7 \\
\hline 6. I'm worried about my future. & 167 & 17.9 \\
\hline $\begin{array}{l}\text { 7. When things get tough, I find it } \\
\text { hard to continue to enjoy life. }\end{array}$ & 157 & 16.8 \\
\hline 8. Difficulties often overwhelm me. & 76 & 8.1 \\
\hline 9. I'm often immersed in anxiety. & 124 & 13.3 \\
\hline 10. I'm not very satisfied with my life. & 104 & 11.1 \\
\hline \multirow[t]{2}{*}{ Depressive symptom } & 184 & 19.7 \\
\hline & 751 & 80.3 \\
\hline
\end{tabular}

Table 3. Selection of items for symptoms of depression $(N=935)$

significant difference in active aging scores among different age groups in this study $(F=1.175, P=0.309)$.

\subsection{Relationship among activities of daily living, symptoms of depression and active aging}

In addition, we analyzed the correlation between active aging and activities of daily living and found a moderate negative correlation ( $r=-0.361)$. Active aging and symptoms of depression also had a moderate negative correlation ( $r=-0.495)$, as show in Table 6. 


\begin{tabular}{lcccc}
\hline Dimensions & Items & Mean scores of each item & Scores from Cao's study ${ }^{12}$ & $t$ \\
\hline \hline Physical vitality & 6 & $3.28 \pm 0.98$ & $3.78 \pm 0.62$ & -15.660 \\
Life satisfaction & 7 & $3.70 \pm 0.78$ & $3.85 \pm 0.48$ & -5.790 \\
Family support & 3 & $4.40 \pm 0.94$ & $3.34 \pm 0.83$ & 34.392 \\
Active participation & 5 & $2.81 \pm 0.95$ & $3.58 \pm 0.75$ & -24.980 \\
Total of PAEQ & 21 & $72.81 \pm 13.63^{*}$ & $77.60 \pm 11.59^{*}$ & -10.744 \\
\hline
\end{tabular}

Note: * $=$ Total average;

$P A E Q$, positive aging evaluation questionnaire.

Table 4 Mean scores of dimensions of PAEQ and comparison $(N=935)$.

\begin{tabular}{|c|c|c|c|}
\hline Variables & PAEQ mean scores & $t / F$ & $P$ \\
\hline \multicolumn{4}{|l|}{ Gender } \\
\hline Male & $73.86 \pm 14.36$ & 2.404 & 0.016 \\
\hline Female & $71.72 \pm 12.76$ & & \\
\hline \multicolumn{4}{|l|}{ Age } \\
\hline $60-69$ & $73.12 \pm 13.52$ & 1.175 & 0.309 \\
\hline $70-79$ & $73.00 \pm 13.88$ & & \\
\hline$\geq 80$ & $71.01 \pm 13.23$ & & \\
\hline \multicolumn{4}{|l|}{ Education } \\
\hline $\begin{array}{l}\text { Primary school } \\
\text { or below }\end{array}$ & $71.34 \pm 13.30$ & 15.348 & $<0.001$ \\
\hline Junior school & $76.28 \pm 13.29$ & & \\
\hline High school & $81.79 \pm 14.44$ & & \\
\hline College or higher & $84.56 \pm 10.42$ & & \\
\hline \multicolumn{4}{|l|}{ Marital status } \\
\hline $\begin{array}{l}\text { Married and with spouse } \\
\text { alive }(A)\end{array}$ & $74.21 \pm 13.246$ & 13.687 & $<0.001$ \\
\hline Single or widowed (B) & $69.65 \pm 13.989$ & & \\
\hline Divorced or separated (C) & $60.86 \pm 11.639$ & & \\
\hline \multicolumn{4}{|l|}{ LSD-t test } \\
\hline A vs. B & & & $<0.001$ \\
\hline A vs. C & & & 0.009 \\
\hline B vs. C & & & 0.088 \\
\hline \multicolumn{4}{|l|}{ Number of children } \\
\hline 0 & $61.50 \pm 15.74$ & 11.484 & $<0.001$ \\
\hline 1 & $73.23 \pm 15.17$ & & \\
\hline $2-3$ & $73.84 \pm 13.34$ & & \\
\hline$\geq 3$ & $71.25 \pm 11.87$ & & \\
\hline \multicolumn{4}{|l|}{ Subjective economic status } \\
\hline Poor & $66.76 \pm 13.29$ & 73.227 & $<0.001$ \\
\hline Fair & $74.25 \pm 12.38$ & & \\
\hline Good & $81.66 \pm 12.35$ & & \\
\hline \multicolumn{4}{|l|}{ Number of chronic diseases } \\
\hline 0 & $81.28 \pm 11.728$ & 61.257 & $<0.001$ \\
\hline 1 & $74.82 \pm 12.83$ & & \\
\hline $2-3$ & $70.71 \pm 12.76$ & & \\
\hline$\geq 3$ & $63.77 \pm 12.19$ & & \\
\hline
\end{tabular}

Note: PAEQ, positive aging evaluation questionnaire.

Table 5. Factors associated with active aging

\begin{tabular}{|c|c|c|c|c|}
\hline Dimensions & $\begin{array}{l}\text { Physical self- } \\
\text { maintenance }\end{array}$ & $\begin{array}{l}\text { Instrumental } \\
\text { activities }\end{array}$ & ADL & DIA-S \\
\hline $\begin{array}{l}\text { Physical } \\
\text { vitality }\end{array}$ & $-0.314^{\star *}$ & $-0.369 * \star$ & $-0.370^{* *}$ & $-0.378^{\star \star}$ \\
\hline $\begin{array}{l}\text { Life } \\
\text { satisfaction }\end{array}$ & $-0.181^{\star *}$ & $-0.283^{\star \star}$ & $-0.264^{\star *}$ & $-0.603^{\star \star}$ \\
\hline $\begin{array}{l}\text { Family } \\
\text { support }\end{array}$ & -0.017 & $-0.066^{\star}$ & $-0.050^{\star *}$ & $-0.188^{\star \star}$ \\
\hline $\begin{array}{l}\text { Active } \\
\text { participation }\end{array}$ & $-0.179 * *$ & $-0.260^{\star \star}$ & $-0.244^{\star *}$ & $-0.147^{\star \star}$ \\
\hline $\begin{array}{l}\text { Total of } \\
\text { PAEQ }\end{array}$ & $-2.74^{\star \star}$ & $-0.377^{\star \star}$ & $-0.361^{\star \star}$ & $-0.495^{\star \star}$ \\
\hline
\end{tabular}

Note: ADL, activity of daily living; DIA-S, depression in old-age scale;

$P A E Q$, positive aging evaluation questionnaire.

${ }^{\star} P<0.05 ;{ }^{*} P<0.01$.

Table 6. Correlation analysis for ADL, DIS-A, and PAEQ $(N=935)$.

\subsection{Multiple linear regression for active aging}

Multiple linear regression models were constructed to evaluate the factors which affect active aging. In the model, total PAEQ score was the dependent variable, whereas gender, education, marital status, number of children, total score of ADL, subjective economic status, depression, and number of chronic diseases were the independent variables. The results showed that depressive symptoms $(\beta=-0.321, P<0.001)$, number of chronic diseases $(\beta=-0.281, P<0.001)$, subjective economic status $(\beta=0.239, P<0.001)$, ability of daily living $(\beta=-0.196, P<0.001)$, education $(\beta=0.126$, $P<0.001)$, number of children $(\beta=0.097, P<0.001)$, and marital status were the influencing factors of active aging in rural elderly. The elderly with no chronic disease, low depression score, good subjective economic status, good ability of daily living, high education level, more children, married and living with spouses had a high level of active aging $(P<0.05)$. These results are presented in Table 7.

\section{Discussion}

In this study, the average score of active aging of rural elderly is 72.81 , which belongs to the upper-middle 


\begin{tabular}{|c|c|c|c|c|c|c|}
\hline \multirow{2}{*}{ Independent variables } & & \multirow{2}{*}{$\beta$} & \multicolumn{2}{|c|}{$95 \% \mathrm{Cl}$} & \multirow{2}{*}{$t$} & \multirow{2}{*}{$P$} \\
\hline & & & LL & UL & & \\
\hline Number of chronic diseases & $\begin{array}{l}0=1 \\
1=2 \\
2-3=3 \\
\geq 3=4\end{array}$ & -0.281 & -4.526 & -3.205 & -11.486 & $<0.001$ \\
\hline Depression symptom & Ture score & -0.321 & -2.413 & -1.763 & -12.612 & $<0.001$ \\
\hline Subjective economic status & $\begin{array}{l}\text { Poor }=1 \\
\text { Fair }=2 \\
\text { Good = } 3\end{array}$ & 0.239 & 3.863 & 5.806 & 9.769 & $<0.001$ \\
\hline Total score of ADL & Ture score & -0.198 & -0.618 & -0.372 & -7.923 & $<0.001$ \\
\hline Education & $\begin{array}{l}\text { Primary school or } \\
\text { below }=1 \\
\text { Junior school }=2 \\
\text { High school }=3 \\
\text { College or higher }=4\end{array}$ & 0.126 & 1.786 & 3.954 & 5.195 & $<0.001$ \\
\hline Number of children & $\begin{array}{l}0=1 \\
1=2 \\
2-3=3 \\
\geq 3=4\end{array}$ & 0.097 & 1.011 & 2.969 & 3.989 & $<0.001$ \\
\hline \multirow[t]{3}{*}{ Marital status } & Married (control group) & & & & & \\
\hline & Divorced or separated & -0.060 & -16.975 & -2.084 & -2.512 & 0.0120 \\
\hline & Single or widowed & -0.102 & -4.501 & -1.654 & -4.242 & $<0.001$ \\
\hline$R^{2}$ & 0.486 & & & & & \\
\hline Adjusted $R^{2}$ & & 0.481 & & & & \\
\hline$F$ & & 109.309 & & & & \\
\hline
\end{tabular}

Note: ADL, activity of daily living; $\mathrm{Cl}$, confidence interval; LL, lower limit; UL, upper limit.

Table 7. Regression analysis of active ageing $(N=935)$

level. The results of this study are similar to those of Jiang's. ${ }^{14}$ This result is closely related to the implementation of the rural medical insurance system, endowment insurance system, social relief system, and other security systems since the founding of new China, which had greatly improved the living standards of the elderly in rural areas. ${ }^{30}$ However, compared with the elderly in urban communities, ${ }^{12}$ the rural elderly showed a relatively low level of activity, especially in the two dimensions of physical vitality and life satisfaction (Table 4). Due to the limitations of rural medical conditions and the generally low level of education of the elderly, rural elderly have lower life satisfaction, self-care ability, and cognitive ability than urban elderly. ${ }^{31-34}$ In the national level, the local financial and health allocation in rural areas is only $53.98 \%$ of that in cities. ${ }^{35,36}$ Therefore, the allocation of rural health institutions, beds, and health technicians is relatively small. ${ }^{35}$ The quality of life of the elderly in rural areas needs further attention and improvement. Health and social service systems in rural areas need to be further strengthened.

Symptoms of depression showed great relevance to active aging in the present study, which is consistent with previous findings. ${ }^{17}$ A good mental state may contribute to more positive attitudes toward health, facilitate functionality and enable the elderly to better integrate themselves. ${ }^{6}$ The prevalence rate of depression among those aged $>60$ years is in the range of $11-57 \%{ }^{37}$ Moreover, the proportion of untreated depressed patients in rural areas is very high - double that in cities. ${ }^{38}$ According to the biological-psychological-social medical model, ${ }^{39}$ health personnel should pay attention not only to the physical diseases of the elderly but also to their psychological problems. In the government's free medical examination program for the elderly, the focus is on the assessment and examination of physiological disease. Psychological problems are ignored. As a result, mental disorders are likely under-recognized and under-treated. This situation suggests that psychological problems should be taken into account when providing health services for the elderly in rural areas.

The number of chronic diseases and ADL are also predictors of active aging. In our study, $79.3 \%$ of the elderly had one or more chronic diseases. This result may be related to the low awareness of disease prevention among the elderly in rural areas. At the same time, it is related to the narrow scope of rural primary health services. Rural primary medical care mostly focuses on the treatment of diseases. However, the publicity of knowledge on disease prevention is relatively little. 
Furthermore, guidance on the rehabilitation of chronic diseases is minimal. Chronic diseases seriously affect people's quality of life due to high morbidity, disability, mortality, and high medical expenses. ${ }^{40-42}$. As chronic diseases cannot be prevented and controlled, the ability of daily life is impaired. In the study, the incidence of elderly people with impaired instrumental daily life ability is relatively high, especially in the use of public vehicles and mobile phones, which may be related to inconvenient rural transportation and underdeveloped information technology. Health is an important pillar of the concept of active aging. ${ }^{1}$ The unhealthy state of the body caused by chronic diseases and the inability to participate in society caused by impaired daily life ability have greatly reduced the active aging level of the elderly in rural areas.

Subjective economic status, education level, marital status, and number of children have certain effects on active aging. This result is consistent with that of a South Korean study. ${ }^{16}$ The self-perception of good economic conditions not only brings self-esteem to the elderly but also makes the elderly more likely to spend money on high-quality food, medical care, and social activities. ${ }^{43}$ Thus, older people can better protect their health, maintain self-esteem at a higher level, and adopt a more positive attitude toward life. A prior study also found that active aging is higher in elderly people with a higher education level. ${ }^{44}$ Elderly people with high education levels in rural areas usually have fixed jobs, retirement pension, wide social contact, and more opportunities to participate in social activities. In addition, their information literacy is also high, and they can effectively access a variety of health resources to ensure their own health. ${ }^{45}$ The arrival of information society makes the realization of active aging inseparable from network technology. The establishment and implementation of information and communication technology training for the elderly can improve the satisfaction and quality of life of participants. ${ }^{46}$

Respondents who have no children, no spouse, and are living with collateral relatives are also the key objects to which medical workers should pay attention. Socio-emotional selectivity theory maintains the focus of older people's social interactions on family relationships to enhance their positive experiences ${ }^{47}$ Influenced by traditional Chinese concepts, the rural elderly put most of their energy into the care of their families, and their emotional support mainly comes from their families. Due to their relatively closed social contacts, rural elderly have higher family needs than urban elderly. ${ }^{48}$ Spouses, children, and other immediate family members are an important source of affection.

Notably, gender and age are not included in the model of influencing factor analysis. No statistical difference was found in the level of active aging between the elderly and the young (Table 5), which is inconsistent with our conventional view. The possible explanations are as follows: (1) since the concept of equality between men and women is deeply rooted in the hearts of the people, elderly women's awareness of participation and health care has gradually improved; and (2) some elderly people were not included in our survey because they could not communicate well for various reasons. This requires further research and exploration.

The influencing factors of active aging were discussed to realize the active aging of the rural elderly at the personal level, and the direction of further efforts was defined to meet the following objectives: (1) increase the construction of the rural environment suitable for the elderly so that the rural elderly have more places for sports and fitness and to provide basic facilities for their physical health; (2) strengthen the mental and physical disease prevention propaganda of prevention and disease screening; (3) provide different security conditions according to different physical conditions of rural elderly in different areas; for example; for the elderly with poor physical condition, the government needs to adopt different forms of old-age support to ensure their dignified life; (4) create opportunities to solve the employment problem of young rural people, help rural entrepreneurs, and encourage more young people be with their parents, which can greatly reduce the labor burden of the rural elderly, enhance emotional exchange between generations, and reduce the occurrence of depression; and (5) facilitate the elderly to engage in literacy activities, not only in writing but also in the application of electronic products. If possible, skills or knowledge training can be carried out for the elderly according to their own conditions.

Active aging is rooted in healthy life and behavior in the pre-old-age period. Attention to active and healthy aging needs to be focused on middle- and young-aged populations. ${ }^{49}$ Chronic diseases, depression, economic status, and education level are not just immediately acquired by the elderly but are accumulated in the young. Therefore, from another aspect, attention should also be paid to the physical health status of the young and middle-aged populations in rural areas to promote the active aging of the elderly in rural areas in the future.

\section{Conclusions}

The study provides a profile of active aging among Chinese rural elders in Xiangtan County. The active aging of the elderly in China's rural areas in Xiangtan County is at a medium-upper level but lower than that of the urban elderly. The regression model indicates that depressive symptoms, ADL, number of chronic diseases, subjective economic status, and education 
are the most important predictors of active aging. The focus group of active aging in rural areas should be elderly people suffering from chronic diseases, physical decline, poor education and economic conditions, childlessness, and who have no partner. These results suggest some tentative recommendations for the elders, primary health personnel, and departments. A more rigorous longitudinal design is necessary to investigate the causality of the related factors and active aging.

\section{Strengths and limitations}

In this study, the active aging questionnaire was used to investigate the active aging status of the elderly in rural areas at the personal level, and relevant information on the status quo and influencing factors of the elderly in rural areas was provided. The results provided not only suggestions for the elderly in rural areas to achieve active aging and improve the quality of life but also direction on the elderly work of primary health workers. The research also provides a certain reference value for the implementation of an active aging policy for the rural elderly.

We affirm that the study has certain limitations. First, the research object only consisted of 9 villages and towns in Xiangtan County, and the results can only have a certain reference value. Second, because it is only a cross-sectional survey, the causal relationship between

\section{References}

1. World Health Organization. Active Ageing A Policy Framework. Madrid, Spain; 2002.

2. Fernández-Ballesteros R, Robine JM, Walker A, Kalache A. Active aging: a global goal. Curr Gerontol Geriatr Res. 2013;2013:298012.

3. Walker A. A strategy for active ageing. Int Soc Secur Rev. 2002;55:121-139.

4. World Health Organization. World Report on Ageing and Health. 2015. https://apps.who.int/iris/ handle/10665/186463. Accessed February 3, 2021.

5. Perales J, Martin S, Luis Ayuso-Mateos J, et al. Factors associated with active aging in Finland, Poland, and Spain. Int Psychogeriatr. 2014;26:1363-1375.

6. Paúl C, Ribeiro O, Teixeira L. Active ageing: an empirical approach to the WHO model. Curr Gerontol Geriatr Res. 2012;2012:382972.

7. Sousa NFDS, Lima MG, Cesar CLG, Barros MBDA. Active aging: prevalence and gender and age differences in a population-based study. Cad Saude Publica. 2018;34:e00173317.

8. Kim J, Lee H, Cho E, Lee KH, Park CG, Cho B. Multilevel effects of community capacity on active various influencing factors and active aging is difficult to explain.

\section{Acknowledgments}

This study received strong support from Xiangtan County Civil Affairs Bureau, Shitan Township Civil Affairs Office, Fenshui Township Civil Affairs Office, and Zhonglupu Township Civil Affairs Office. We thank all the research participants for their kind participation.

\section{Ethical approval}

This study was approved by the Ethics Review Committee of Behavioral Medicine and Nursing Research of Xiang-ya Nursing School, South-Central University China (No. 2019013). All the participants and their families gave their informed consent before the survey. The researcher also emphasized to potential participants that they had the right to refuse to participate or to stop participating at any time. The questionnaires were recorded anonymously, and no information regarding identity was collected.

\section{Conflicts of interest}

All contributing authors declare no conflicts of interest. aging in community-dwelling older adults in South Korea. Asian Nurs Res. 2020;14:36-43.

9. Hsu H, Liang J, Luh D, Chen C, Wang Y. Social determinants and disparities in active aging among older Taiwanese. Int J Environ Res Public Health. 2019;16:3005.

10. Haque MN. Active ageing level of older persons: regional comparison in Thailand. J Aging Res. 2016;2016:9093018.

11. Salazar-Barajas ME, LilloCrespo M, Hernandez CP, et al. Factors contributing to active aging in older adults, from the framework of Roy's adaptation model. Investig Educ Enferm. 2018;36:e8.

12. Wenting $C$, Changjuan $Z$. Analysis on the active aging status and influencing factors of some resident elderly in Shanghai. Chin $J$ Gerontol. 2019;39:2538-2541.

13. Wenjuan S. Study on the Active Aging of the Elderly and the Relation between the Active Aging and Life Style. Beijing: Beijing Sport University; 2019.

14. Xingli J, Donghua W. Current status of active aging of 381 rural seniors and its influence factors. J Nurs. 2019;26:41-45. 
15. Thanakwang K, Isaramalai SA, Hatthakit U. Development and psychometric testing of the active aging scale for Thai adults. Clin Interv Aging. 2014;9:1211-1221.

16. Pham VT, Chen YM, Van Duong T, Nguyen T, Chie WC. Adaptation and validation of active aging index among older Vietnamese adults. J Aging Health. 2020;32:604-615.

17. Paúl $C$, Teixeira L, Ribeiro $O$. Active aging in very old age and the relevance of psychological aspects. Front Med (Lausanne). 2017;4:181.

18. Xuelian F, Yinli S, Yang G, Liqiong L. Correlation between active aging and depression in the elderly. Med Philos (A). 2018;39:37-39.

19. Jensen L, Monnat SM, Green JJ, Hunter LM, Sliwinski MJ. Rural population health and aging: toward a multilevel and multidimensional research agenda for the 2020s. Am J Public Health. 2020;110:1328-1331.

20. Peltzer K, Phaswana-Mafuya N, Pengpid S. Ruralurban health disparities among older adults in South Africa. Afr J Prim Health Care Fam Med. 2019;11:e1-e6.

21. Shi C, Hu B. Preferences for formal social care in rural and urban China: evidence from a national survey. J Gerontol Soc Work. 2020;63:19-40.

22. Xiangtan Bureau of Statistics. National economic and social development statistics bulletin of Xiangtan City in 2018, 2019. http://tjj.hunan.gov.cn/tjfx/ tjgb/szgb/xts/201904/t20190416_5316619.html. Accessed February 3, 2021.

23. Bo Y. Research on the Problem of Providing for the Aged in Rural Areas of Qinhuangdao City. Qinhuangdao: Hebei Normal University of Science and Technology; 2018 (in Chinese).

24. Min H. Evaluation Study of the Elderly Active Aging. Nanchang: Nanchang University; 2012 (in Chinese).

25. Lawton MP, Brody EM. Assessment of older people: self-maintaining and instrumental activities of daily living. Gerontologist. 1969;9:176-186.

26. Heidenblut S, Zank S. Development of a new screening instrument for geriatric depression. The depression in old age scale (DIA-S). Z Gerontol Geriatr. 2010;43:170-176.

27. Rashedi V, Rezaei M, Foroughan M, Delbari A. Validity and reliability of the depression in old age scale (DIA-S) in Iranian older adults. Arch Gerontol Geriatr. 2016;66:193-197.

28. Heidenblut S, Zank S. Screening for depression with the Depression in Old Age Scale (DIA-S) and the Geriatric Depression Scale (GDS15). GeroPsych. 2014;27:41-49.
29. Li GP, Liu H. Reliability and validity of the Chinese version of the depression in old age scale (DIA-S). Mod Prev Med. 2019;46:1541-1544 (in Chinese).

30. Yuanzheng Y. Social welfare for the aged and patients - retrospect and prospect of China's rural medical and endowment insurance system in the past 70 years. J Soc Dev. 2019;6:185-203 (in Chinese).

31. Yanan C. Influential Factors of life Satisfaction of the Elderly and their Urban-Rural Differences - An Empirical Analysis based on CHARLS 2015 Data. Hangzhou: Zhejiang University of Finance \& Economics; 2018 (in Chinese).

32. Wei M, Wang JW. Gender and urban - rural differences in cognitive function degradation of the elderly in China. Chin Mental Health J. 2019;33:950-954 (in Chinese).

33. Ge D, Chu J, Zhou C, Qian Y, Zhang L, Sun L. Rural-urban difference in the use of annual physical examination among seniors in Shandong, China: a cross-sectional study. Int J Equity Health. 2017;16:86.

34. Ruiling Z. A study on the life satisfaction of the rural elderly: based on data from Henan Province. Stat Inf Forum. 2018;33:114-120.

35. Center for Health Statistics and Information. An Analysis Report of National Health Services Survey in China, 2013. http://www.nhc.gov.cn/ewebeditor/uploadfile/2016/10/20161026163512679.pdf. Accessed February 3, 2021.

36. Maihong $\mathrm{H}$. Research on Health Service Equity in China. Chongqing: Third Military Medical University; 2013 (in Chinese).

37. Chen Y, Hicks A, While AE. Depression and related factors in older people in China: a systematic review. Rev Clin Gerontol. 2012;22:52-67.

38. World Health Organization. China Country Assessment Report on Ageing and Health. Geneva; 2016.

39. Engel GL. The need for a new medical model: a challenge for biomedicine. Science. 1977;196: 129-136.

40. Mengming L. Research of Protective Factors of Resilience in Elderly Patients with Chronic Diseases. Tangshan: North China University of Science and Technology; 2019 (in Chinese).

41. Song XM, Zhou YY, Guo P, Feng SW, Xue SQ. An analysis on disabling impacts of chronic diseases in Chinese elderly. Popul Dev. 2016;22:79-83 (in Chinese).

42. Yokota RT, Berger N, Nusselder WJ, et al. Contribution of chronic diseases to the disability burden in a population 15 years and older, Belgium, 19972008. BMC Public Health. 2015;15:229. 
43. Wang R, Feng Q, Dupre ME, et al. Objective and subjective financial status and mortality among older adults in China. Arch Gerontol Geriatr. 2019;81:182-191.

44. Kim KS, Kim SY. Multi-dimensional measuring and multi-level analysis of active ageing in 16 OECD countries. Soc Welfare Pol. 2014;41:1e-32e.

45. Tennant B, Stellefson M, Dodd V, et al. eHealth literacy and web 2.0 health information seeking behaviors among baby boomers and older adults. J Med Internet Res. 2015;17:e70.

46. Díaz-López Mdel P, López-Liria R, Aguilar-Parra JM, Padilla-Góngora D. Keys to active ageing: new communication technologies and lifelong learning. SpringerPlus. 2016;5:768.

47. Carstensen LL, Turan B, Scheibe S, et al. Emotional experience improves with age: evidence based on over 10 years of experience sampling. Psychol Aging. 2011;26:21-33.

48. Gui Y. Study on Differences in Urban and Rural Psychological Needs for the Elders. Changsha: Hunan Normal University; 2014 (in Chinese).

49. Guangzong M. Health for all regardless of age: healthy aging from the perspective of aging. Popul Dev. 2018;24:11-13 (in Chinese). 